\title{
The second enzyme in pyrrolnitrin biosynthetic pathway is related to the heme-dependent dioxygenase superfamilyt
}

\author{
Walter De Laurentis $\ddagger$, Leang Khim ${ }^{\ddagger}$, J.L. Ross Anderson $§$, Ariane Adam ${ }^{\square}$, Kenneth A. \\ Johnson, Robert S. Phillips ${ }^{\wedge}$, Stephen K. Chapman ${ }^{\S}$, Karl-Heinz van Pee ${ }^{\square}$, and James H. \\ Naismith ${ }^{\ddagger},{ }^{*}$ \\ Centre for Biomolecular Sciences, EastChem, The University, St Andrews, Scotland, KY16 9ST, \\ U.K., Department of Chemistry, EastChem, The University of Edinburgh, Scotland EH9 3JJ; \\ Biochemie, Technische Universität Dresden, D-01062 Dresden, Germany and Department of \\ Chemistry, Biochemistry and Molecular Biology, University of Georgia, Athens Georgia 30602
}

\section{Abstract}

Pyrrolnitrin is a commonly used and clinically effective treatment for fungal infections and provides the structural basis for the more widely used fludioxinil. The pyrrolnitrin biosynthetic pathway consists of four chemical steps, the second of which is the rearrangement of 7-chlorotryptophan by the enzyme PrnB, a reaction that is so far unprecedented in biochemistry. When expressed in Pseudomonas fluorescens, PrnB is red in color due to the fact that it contains 1 mole of heme $b$ per mole of protein. The crystal structure unexpectedly establishes PrnB as a member of the heme-dependent dioxygenase superfamily with significant structural but not sequence homology to the two-domain indoleamine 2,3-dioxygenase enzyme (IDO). The heme-binding domain is also structurally similar to that of tryptophan 2,3-dioxygenase (TDO). Here we report the binary complex structures of PrnB with D- and L-tryptophan and D- and L-chloro-tryptophan. The structures identify a common hydrophobic pocket for the indole ring but exhibit unusual heme ligation and substrate binding when compared with that observed in the TDO crystal structures. Our solution studies support the heme ligation observed in the crystal structures. Purification of the hexahistidine-tagged PrnB yields homogeneous protein that only displays in vitro activity with 7-chloro-L-tryptophan after reactivation with crude extract from the host strain, suggesting that an as yet unknown cofactor is required for activity. Mutation of the proximal heme ligand results, not surprisingly, in inactive enzyme. Redox titrations show that PrnB displays a significantly different reduction potential to that of IDO or TDO, indicating possible differences in the PrnB catalytic cycle. This is confirmed by the absence of tryptophan dioxygenase activity in PrnB, although a stable oxyferrous adduct (which is the first intermediate in the TDO/IDO catalytic cycle) can be generated. We propose that PrnB shares a key catalytic step with TDO and IDO, generation of a tryptophan hydroperoxide intermediate, although this species suffers a different fate in PrnB, leading to the eventual formation of the product, monodechloroaminopyrrolnitrin.

Biosynthetic pathways in bacteria remain a major source of interest primarily due to the value of the final natural product but often for the novel chemistry contained within them.

\footnotetext{
${ }^{\dagger}$ This work was supported by the Biotechnology and Biological Sciences Research Council (UK) through a grant to JHN.

" To whom correspondence should be addressed: Centre for Biomolecular Sciences, The University, St Andrews, KY16 9ST UK. Phone: 44-1334-463792 Fax 44-1334-467229 naismith@st-and.ac.uk.

tUniversity of St Andrews

§University of Edinburgh

$\square$ University of Dresden

University of Georgia

The first two authors made an equal contribution
} 
The pyrrolnitrin biosynthetic pathway is an example of such a pathway in which the final product, a potent antifungal compound used to treat skin infections, is produced by a succession of elegant transformations whose mechanisms are not immediately obvious. This pathway is common to many organisms such as Enterobacter agglomerans (1), Myхосоccus fulvus (2), Corallococcus exiguous (2), Cystobacter ferrugineus (2) and Serratia spp (3) and was first isolated and studied in Pseudomonas species. Through both genetic engineering of plants to include the pyrrolnitrin biosynthetic gene cluster and direct addition of Pseudomonas spp to plants, it has been demonstrated that fungal moulds can be effectively controlled (4-6). Pyrrolnitrin is believed to exert its antifungal activity through inhibition of the respiratory electron transport system (7).

Previous knockout studies in Pseudomonas fluorescens have identified the intermediate products from each of the biosynthetic proteins in the pyrrolnitrin pathway and some of the transformations have been well characterized (8). The first step is the regioselective incorporation of chlorine into D- and L- tryptophan by PrnA. The crystal structure and subsequent solution studies indicate that $\mathrm{HOCl}$ is formed at the FAD cofactor and passed down a channel towards the substrate (9) where a $\mathrm{N}$-chlorolysine species is formed prior to the chlorination of tryptophan (10). A similar mechanism is likely to operate in the third step, as PrnC, which is known to have significant sequence homology to PrnA, chlorinates monodechloroaminopyrrolnitrin. The final step, in which the amine of the substrate is oxidized to a nitro group, is carried out by the non-heme iron and iron-sulfur clustercontaining PrnD and has also been recently examined (11).

Although the product and substrate of PrnB are known, the enzyme has to date received little attention. Interestingly, both D- and L-tryptophan can be incorporated into the final product of the complete pathway, pyrrolnitrin, and it has been shown that PrnA can utilize either of these enantiomers as its substrate. This indicates that PrnB utilizes both enantiomers of 7-chlorotryptophan alternatively an as yet unidentified racemase interconverts the D- and L- isomers, with PrnB only acting on one. Only 7-chloro-Ltryptophan is isolated from the Pseudomonas fluorescens mutant strain lacking PrnB, suggesting this is preferred natural enantiomer (8).

Interest in tryptophan biochemistry is dominated by the study of its degradation in the kynurenine pathway by the enzymes tryptophan 2,3-dioxygenase (EC 1.13.11.11, TDO) and indoleamine 2,3-dioxygenase (EC 1.13.11.52, IDO). Both are b-type heme dependent dioxygenases and catalyse the conversion of L-tryptophan to L-formylkynurenine by incorporating two atoms of molecular oxygen into the indole ring of the substrate (Scheme 2). TDO is a tetrameric enzyme containing four molecules of heme b per tetramer $(12,13)$ and IDO is a monomeric, two-domain protein containing one molecule of heme b per protein molecule (14). The heme group is found enclosed and in the presence of substrate free from water (12). Given the similarity in the heme-binding domains of the two proteins and in their active sites, it seems likely that both use a similar mechanism, although IDO has broader substrate specificity and has been shown to perform a more versatile role in vivo. Two mechanisms have been proposed: the Criegee mechanism involves the formation of a tryptophan hydroperoxide intermediate that undergoes a Criegee-type rearrangement (12, 13); the other mechanism involves the formation of a four-membered dioxetane ring (14). In comparison to the mechanism operating in $\operatorname{PrnB}$, there must be significant differences as the products are dissimilar, although both result in the opening of the indole ring of tryptophan. Most significantly for PrnB, there is no incorporation of oxygen into the product. There is no overall significant sequence similarity of PrnB to either IDO or TDO, hinting at a novel mechanism for the formation of monodechloroaminopyrrolnitrin. Recently, another member of the tryptophan dioxygenase family was isolated from Shewanella oneidensis which shares 
the same fold as IDO, contains heme b but is not active with tryptophan and contains a highly divergent sequence from other members of the family (PDB 2NWB).

In this paper we report the initial structural and spectroscopic characterization of the 361 residue, $43 \mathrm{kDa}$ PrnB protein from Pseudomonas fluorescens BL915. We have determined the X-ray crystal structure using multiple wavelength anomalous scattering in the presence of its putative substrates (7-chloro-D-tryptophan and 7-chloro-L-tryptophan) and its substrate analogues D- and L- Tryptophan. These structural data show striking similarities between PrnB and both IDO (14) and TDO $(12,13)$.. Our spectroscopic studies reveal significant differences between PrnB and IDO/TDO, suggesting that PrnB does indeed utilize a novel mechanism.

\section{Materials and Methods}

\section{Purification of PrnB}

The cloning, purification and crystallization of PrnB have already been reported in detail (15). We were unable to obtain folded soluble protein from any Escherichia coli system but instead relied upon the Pseudomonas fluorescens expression system. Native protein had a tendency to aggregate and a triple mutant (C21S, C60S and C185S) was also used. The protein had a non-cleavable His tag at the $\mathrm{N}$-terminus. Briefly, $P$. fluorescens cells with a plasmid containing the his tagged $p r n B$ gene were grown. After centrifugation, the cells were washed with $0.1 \mathrm{M}$ potassium phosphate buffer ( $\mathrm{pH}$ 7.0) and then one part of cells was resuspended in two parts of potassium phosphate buffer ( $\mathrm{pH}$ 7.0) and the cells were disrupted by sonication for $3 \mathrm{~min}$ (Branson Sonifier, with $5 \mathrm{~s}$ pulse, $10 \mathrm{~s}$ break, $0{ }^{\circ} \mathrm{C}, 65 \%$ amplitude). After removal of the cell debris by centrifugation, the crude extract was either stored at $-20{ }^{\circ} \mathrm{C}$ or immediately used for purification of the enzyme. In the later case $3 \mathrm{ml}$ aliquots of the crude extract were loaded onto a Ni-chelating sepharose fast flow column (1 $\times 7 \mathrm{~cm}, 0.2 \mathrm{ml} / \mathrm{min}$ ) equilibrated with $50 \mathrm{mM}$ Tris phosphate buffer ( $\mathrm{pH} 8.0$ ) containing 150 $\mathrm{mM} \mathrm{NaCl}$ and $20 \mathrm{mM}$ imidazole. After removing all non-bound protein by washing the column with $40 \mathrm{ml}$ of the same buffer, proteins were eluted with a linear gradient over a period of $500 \mathrm{~min}(0.2 \mathrm{ml} / \mathrm{min})$ from $150 \mathrm{mM} \mathrm{NaCl}, 20 \mathrm{mM}$ imidazole in $50 \mathrm{mM}$ Tris phosphate buffer (pH 8.0) to $300 \mathrm{mM} \mathrm{NaCl}, 500 \mathrm{mM}$ imidazole in $50 \mathrm{mM}$ Tris phosphate buffer ( $\mathrm{pH}$ 8.0) with the collection of $2 \mathrm{ml}$ fractions. Fractions were analyzed by SDS-PAGE and those containing PrnB were pooled and dialyzed against $10 \mathrm{mM}$ Tris phosphate buffer (pH 8.0) overnight. The purified protein (mutant and native) was a deep red color characteristic of heme containing enzymes.

\section{Structural Biology}

The protein was characterized by mass spectrometry including acetone extraction of lyophilized sample. Mass spectrometric analysis of the acetone extraction is consistent with the presence of heme b (peak at $615.95 \mathrm{~nm}$; calculated $615.6 \mathrm{~nm}$ ). Protein crystals were only obtained from the triple mutant protein and the details of the crystallization have been reported (15). The best results were obtained using with $1.5 \mu$ l protein solution $(16 \mathrm{mg} / \mathrm{ml}$ $50 \mathrm{mM}$ Tris $\mathrm{pH} 7.5)$ plus $1.5 \mu \mathrm{l}$ of precipitant ( $0.22 \mathrm{M}$ magnesium sulfate, $16 \% \mathrm{w} / \mathrm{v}$ PEG 3350) irrespective of whether protein was incubated with tryptophan (as a saturated solution) or not. We first recorded a data set to $1.75 \AA$ resolution of protein crystallized in the presence of L-tryptophan. We attempted to solve the structure with molecular replacement using apo TDO from Xanthomonas campestris (PDB 1ZEE) but were unable to find a solution. This was unsurprising given the less than $20 \%$ identity and we resorted to experimental phasing. We collected data on a second crystal from the same crystallization conditions at three wavelengths to a resolution of $2.0 \AA$ chosen to optimize the anomalous $(1.739 \AA)$ and dispersive differences $(1.741 \AA$ and $1.722 \AA$ ) resulting from the iron. This 
crystal, although grown in the same conditions, had a different unit cell and spacegroup. SOLVE located the single iron and density modification allowed us to quickly trace a monomer. We then extended the phases to $1.75 \AA$ with data collected with a wavelength of $1.49 \AA$. This structure was used to solve the structure of the protein crystallized with Dtryptophan. Protein crystallized with a racemic mixture of D and L 7-chlorotryptophan under the same conditions gave a $2.0 \AA 7$-Cl-D-trptophan complex structure. Protein crystallized with pure 7-Cl-L-tryptophan, synthesized as previously reported(16), gave a $2.4 \AA$ 7-Cl-Ltryptophan complex structure. The structures were refined using REFMAC5 (17). Ligands were added only when density was clear. The ligand dictionaries were taken from the REFMAC defaults. TLS parameters were employed in refinement, we investigated the use of anisotropic thermal factor refinement but for the two higher resolution data sets, the improvement in Rfree was less than $0.5 \%$ and so isotropic thermal factors were used. Full crystallographic details are given in Table 1.

\section{Activity assay}

Cultures of Pseudomonas fluorescens BL915 $\Delta$ orf1-4 which had been engineered to lack the pyrrolnitrin pathway but harbored a plasmid containing $p r n B$ or mutants of $p r n B$ were grown overnight in HNB medium. The cultures were diluted 1 to 20 in fresh medium and cells were grown for a further $24 \mathrm{hrs}$ at $30^{\circ} \mathrm{C}$ and $200 \mathrm{rpm}$ on orbital shaker incubators. To some of the cultures sterile filtered 7-Cl-tryprophan (racemic) was added to a final concentration of $1 \mathrm{mM}$. Cells were grown for additional 2 days. $20 \mathrm{ml}$ of the cultures with or without 7-Cl-tryptophan were extracted twice with one volume of ethyl acetate. The extracted mixtures were dried in vacuo and redissolved in $130 \mu \mathrm{l}$ methanol. The extracts were analyzed by thin layer chromatography on silica plates using toluene/dioxin (9:1) as the eluent and by isocratic HPLC with methanol/ $\mathrm{H}_{2} \mathrm{O}(65: 35)$ as the eluent, using a Varian OmniSpher or Lichrosphere 100, RP-18, $5 \mu \mathrm{m}, 250 * 4.6 \mathrm{~mm}$ column. Flow rate was $1 \mathrm{ml} /$ min and compounds were detected at 220 and $303 \mathrm{~nm}$. In vitro activity was analyzed by incubating $225 \mu \mathrm{l}$ PrnB-containing protein solution with $125 \mu \mathrm{l} 1 \mathrm{mM}$ 7-chlorotryptophan (racemic or pure enantiomers) and $150 \mu \mathrm{l} 0.1 \mathrm{M}$ potassium phosphate buffer (pH 7.0) at 30 ${ }^{\circ} \mathrm{C}$ for $16 \mathrm{~h}$. The assay mixtures were extracted twice with $500 \mu \mathrm{l}$ ethyl acetate and the combined organic extracts were dried in vacuo and re-dissolved in $130 \mu \mathrm{l}$ methanol and 20 $\mu \mathrm{l}$ of the resulting solution were used for HPLC analysis as described above. For reactivation of PrnB after dialysis or purification by immobilized nickel-chelating affinity chromatography, $225 \mu \mathrm{l}$ of PrnB-containing protein solution were incubated with $125 \mu \mathrm{l} 1$ $\mathrm{mM}$ 7-chlorotryptophan, $150 \mu \mathrm{l} 0.1 \mathrm{M}$ potassium phosphate buffer (pH 7.0), and $150 \mu \mathrm{l}$ of cell-free crude extract from the host strain P. fluorescens BL915 $\Delta$ orf1-4 at $30^{\circ} \mathrm{C}$ for $16 \mathrm{~h}$ and analyzed by HPLC as described above.

\section{Magnetic Circular Dichroism (MCD) Spectroscopy}

MCD spectra were recorded using Jasco J810 and J730 models for the UV-vis and near-IR regions, respectively. An Oxford Instruments SM-1 superconducting solenoid with a $25 \mathrm{~mm}$ room-temperature bore was used to generate a $6 \mathrm{~T}$ magnetic field. MCD intensities are linear with respect to the magnetic field at room temperature and are plotted normalized to magnetic field as $\Delta \varepsilon / H\left(\mathrm{M}^{-1} \mathrm{~cm}^{-1} \mathrm{~T}^{-1}\right)$. Samples were prepared in deuterated Hepes buffer $(50 \mathrm{mM})$ at $\mathrm{pH} 7.5$.

\section{Electron Paramagnetic Resonance (EPR) Spectroscopy}

X-Band EPR spectra $(10 \mathrm{~K})$ were recorded on a Bruker ER-300D series electromagnet and microwave source interfaced to a Bruker EMX control unit and fitted with an ESR-9 liquid Helium flow cryostat from Oxford Instruments and a dual mode microwave cavity from Bruker (ER-4116DM). Quantification of $S=1 / 2$ species was carried out by comparison with the spin standard $\mathrm{Cu}^{\mathrm{II}}$ (EDTA) using the method of Aasa and Vanngard (18). Magnetic field 
magnitude is detected by a Hall probe within the cavity of the instrument. Additionally, the $g$ values of the $\mathrm{Cu}^{\mathrm{II}}$ EDTA standard $(g=2.32$ and 2.075$)$ were used to ensure the correct field values for the detected frequency (observed values identical to those given above). Frequency is monitored within the EMX unit and also with an additional frequency counter attached to the microwave source.

\section{OTTLE Potentiometry}

Anaerobic potentiometric titrations were carried out as described previously(19), at $25^{\circ} \mathrm{C}$ using a modified quartz EPR OTTLE cell. A range of mediators were added (Flavin mononucleotide (-200 $\mathrm{mV}$ vs SHE), 2-hydroxy-1,4-naphthoquinone (-145 mV), 5hydroxy-1,4-naphthoquinone $(-3 \mathrm{mV})$, phenazine ethosulfate $(+55 \mathrm{mV})$, phenazine methosulfate $(+82 \mathrm{mV}), 1,2$-naphthoquinone $(+135 \mathrm{mV}))$ to ensure efficient reduction and reoxidation. Titrations were performed both in the absence and presence of L-tryptophan and D-tryptophan $(15 \mathrm{mM})$ and the heme reduction potentials were determined by fitting the data to the Nernst equation for a single electron process using the Microcal Origin software. Reduction potentials are quoted versus the standard hydrogen electrode (SHE).

\section{Electronic spectroscopy and dissociation constant measurements}

Electronic absorption spectra were recorded using a Cary 50-Probe UV-Visible spectrophotometer at $25^{\circ} \mathrm{C}$. Dissociation constants $\left(K_{\mathrm{d}}\right)$ were determined by established spectrophotometric procedures. The spectrum of the oxyferrous species was recorded using an Applied-Photophysics stopped-flow spectrophotometer (SX.17MV) in conjunction with a diode array detector, housed in an anaerobic glove box (Belle Technology, $\left[\mathrm{O}_{2}\right]<5 \mathrm{ppm}$ ). A solution of pre-reduced $\operatorname{PrnB}(40 \mu \mathrm{M})$ was mixed with oxygenated buffer at $15{ }^{\circ} \mathrm{C}$ and spectra were recorded using the diode array detector.

\section{Results}

\section{Structure of PrnB}

The asymmetric unit of all crystals contains a monomer of the protein. We investigated crystal contacts for any dimerization using the Protein Interaction server (PISA)(20) at the European Bioinformatics Institute (EBI) and this indicates the protein is a monomer, consistent with gel filtration results. The final refined structure consists of residue 5 to 361 (true C-terminus); one loop of 9 residues which connects two a helices (323 to 331) is disordered. The $\mathrm{N}$-terminal tag (40 residues) is not seen in any structure. The structure contains $17 \mathrm{a}$ helices connected together by a series of loops; there are two short $\beta$-strands in the structure (Figure 1). The experimental electron density map showed clear density for a heme group in each of the five structures. The molecule can be split into two domains, a small N-terminal domain of 120 amino acids (a 1-7) and the core helical domain of 240 amino acids. The helical core domain is related to myoglobin. The structure is very similar to that seen for IDO (PDB 2D0T) (root mean square deviation of $2.4 \AA$ for $268 \mathrm{Ca}$ atoms) and the core helical domain is topologically identical to TDO (PDB 2NW8) (root mean square deviation of $3.2 \AA$ for $168 \mathrm{Ca}$ atoms). There are differences in detail regarding the loops and positioning of a helices that explain why molecular replacement failed to work. The three cysteine residues that were mutated are remote from the heme. However, despite being grown in the presence of L-tryptophan, only a water molecule is found at the heme in the $1.75 \AA$ structure. The heme is bound in a solvent exposed cleft with 7-propionate group accessible and distal face exposed. The 6-propionate group makes a salt contact with R310. The heme is also anchored to the protein by a number of van der Waal interactions with residues on helices a 8, a 9, a 10 and a 16 which pack around the distal face and hydrophobic edge of the porphoryin ring. The precise degree of solvent accessibility of the distal face of the heme must be treated with caution as the disordered loop is adjacent to heme cleft and 
could fold over sealing the cleft. The proximal ligand to the heme iron is His 313 which is located on a 16. The distal face of the porphyrin has a large hydrophobic cavity above it. Calculations using CASTP (21) estimate the surface area of the cavity as $480 \AA^{2}$ with a volume of $580 \AA^{3}$ in the substrate free structure. The distal ligand to the iron in this structure is a water molecule which is hydrogen bonded to a second water molecule which is in turn bound to the main chain of protein. Strangely, a crystal grown under essentially the same conditions and used in MAD phasing does unambiguously have L-tryptophan bound at the active heme. We have no explanation for this, nor have we been able to obtain good quality crystals of the substrate free form again. Crystals grown in the presence of D-tryptophan clearly show D-tryptophan bound as do crystals grown with 7-Cl-L-tryptophan. The presence or absence of a ligand at the heme does not significantly alter the PrnB structure. The changes are displacement of water molecules and movement of a few flexible side chains.

In all four complex structures, the indole is bound in the hydrophobic pocket above the distal face and the main chain is located at the iron. However, we see two modes of binding of the indole ring and two modes of binding of the main chain; in total three distinct complexes (the D-tryptophan and 7-Cl-D-tryptophan complexes are essentially identical, the only difference being the additional chlorine atom on the ligand). In the 7-Cl-L-tryptophan and 7-Cl-D-tryptophan (and D-tryptophan) complexes the indole rings essentially superimpose. The indole ring is sandwiched between the side chains of L140 and Phe 201 on one side and the main chain between G223 to V225 on the other. The five membered ring of the indole system is capped by Y209 and the chlorine atom is found in a pocket bounded by V117, P222 and is $3.8 \AA$ from a water molecule. In the L-tryptophan complex, the indole ring is rotated relative to other three complexes, in such a way that the 6 membered ring overlap but the five membered rings are on opposite sides (Figure 2). In the L-tryptophan complex, the indole nitrogen points towards the side chain of $\mathrm{S} 143$ (>4.0 $\mathrm{\AA}$ ).

Despite the difference in the indole ring location between the L-tryptophan and 7-Cl-Ltryptophan complexes, the main chain of the tryptophans does superimpose. The amino nitrogen atom of the tryptophan is ligated to the iron and the carboxylic acid points into an oxyanion hole G223-V225; one oxygen atom makes a hydrogen bond to the backbone amide of V225 and the other makes hydrogen bonds to water molecules. In the 7-Cl-D-tryptophan complex, one of the oxygen atoms of the carboxyl group is bound to the iron, with the other oxygen making the same hydrogen bond to V225 as seen for the L enantiomers, the amino nitrogen makes hydrogen bonds to water molecules.

\section{Activity of PrnB}

The native enzyme and the triple cysteine his-tagged mutant used for the biophysical characterization behave identically. The cell-free extracts containing PrnB showed a spot on silica thin layer plates with an $\mathrm{R}_{\mathrm{f}}$-value of 0.24 that reacted with van Urk's reagent (reacts with indoles and their derivatives) to give a pink color immediately. This turns olive with time and is thought to correspond to 2-( $2^{\prime}$ aminophenyl)pyrrole. This is the product one would expect from the reaction of PrnB with tryptophan. Van Urk's reagent is made by dissolving $1 \mathrm{~g}$ of p-dimethylaminobenzaldehyde in $50 \mathrm{ml}$ of $36 \% \mathrm{HCl}$ followed by the addition of $50 \mathrm{ml}$ ethanol; the solution is stored at $4{ }^{\circ} \mathrm{C}(8)$. HPLC-analysis of cell free extracts shows that a peak at 6 min is eluted that exhibits a UV-spectrum with a maximum at $303 \mathrm{~nm}$. Mass spectrometry of the peak reveals a mass of $159\left(\mathrm{M}+\mathrm{H}^{+}\right)$, consistent with 2( 2 ' aminophenyl)pyrrole. Extracts of culture medium of cells fed with 7-Cl-tryptophan show a spot on thin layer plates with an $\mathrm{R}_{\mathrm{f}}$-value of 0.5 that stains as described above when sprayed with van Urk's reagent. HPLC analysis of cell free extracts of the 7-Cl-tryptophan fed cells shows a peak at $10.5 \mathrm{~min}$. The sample corresponding to this peak, when analyzed by mass spectrometry, showed a parent ion peak of 193/195 amu with a ratio of 3:1; 
expected for monodechloroaminopyrrolnitrin (PrnB product, Scheme 1). The same peak was obtained when crude cell-free extract was incubated in the presence of racemic 7-

chlorotryptophan (Figure 3) as well as the pure D- and L-enantiomers (Figure 3). The yield of monodechloroaminopyrrolnitrin with 7-chloro-L-tryptophan is about 1.5 fold higher than with the D-enantiomer. When the crude extract was dialyzed overnight or purified by $\mathrm{Ni}$ chelating chromatography, no PrnB activity is detectable. However, when crude extract of the host strain which did not contain PrnB (and has no PrnB activity) was added to the dialyzed PrnB full activity was restored (Figure 3). In vitro analysis of the activity of PrnB with L- and D-tryptophan could not be performed since both cell-free extracts (the PrnBcontaining one and the one from the host strain) already contain L-tryptophan which cannot be removed without inactivation of the enzyme. The H313A mutant had no activity. Attempts to purify the mutant resulted in a colorless (presumably heme free) aggregated protein. We assayed for TDO activity by looking for the formation of $\mathrm{N}$-formylkynurenine at $321 \mathrm{~nm}$. The oxyferrous form of the enzyme was generated and titrated against Ltryptophan in a multi-mixing experiment in the stopped-flow. There was no evidence of reaction either by monitoring for $\mathrm{N}$-formylkynurenine formation or spectral changes at the heme (versus control experiments in the absence of L-Trp).

\section{Spectroscopy of PrnB}

UV visible spectroscopy shows the characteristic signature of heme-binding proteins with the $\mathrm{Fe}^{3+}$ peak at $405 \mathrm{~nm}$ and the $\mathrm{Fe}^{2+}$ peak at $417 \mathrm{~nm}$. The ligand titration experiments give a $\mathrm{K}_{\mathrm{D}}$ of $60 \mu \mathrm{M}$ for L-tryptophan, $39 \mu \mathrm{M}$ for D-tryptophan and $4.9 \mu \mathrm{M}$ for 7-Cl-L-tryptophan binding to $\mathrm{Fe}^{3+} \mathrm{PrnB}$. We do not have sufficient quantities of pure 7-Cl-D-tryptophan for spectroscopic study. Due to poor solubility at higher concentrations, it was impossible to directly measure $\mathrm{Kd}$ values for tryptophan with $\mathrm{PrnB}$ in the reduced, $\mathrm{Fe}^{2+}$ form. However, using our electrochemical data we can estimate these values to be as follows: $11 \mathrm{mM} \pm-2$ $\mathrm{mM}$ for L-Trp; $40 \mathrm{mM} \pm 7 \mathrm{mM}$ for D-Trp and $140 \mu \mathrm{M} \pm 20 \mathrm{uM}$ for 7-Cl-Ltryptophanopop. The $\mathrm{K}_{\mathrm{D}}$ of L-tryptophan and 7-Cl-L-tryptophan with the $\mathrm{Fe}^{3+}$ form are very sensitive to $\mathrm{pH}$ and titrate with a $\mathrm{pKa}$ of around 6.5. The midpoint reduction potential of PrnB in the absence of ligand is $-109 \mathrm{mV}$. The presence of ligand significantly alters the redox potential of PrnB, $-287 \mathrm{mV}$ (L-tryptophan), $-243 \mathrm{mV}$ (D-tryptophan) and $-195 \mathrm{mV}$ (7-Cl-L-tryptophan) respectively (Figure 4). In the $\mathrm{Fe}^{2+}$ state, in the absence of substrate, PrnB readily forms an oxyferrous complex. The decay of this complex by autooxidation has a rate constant of $0.0015 \mathrm{~s}^{-1} \pm 0.0001 @ 15^{\circ} \mathrm{C}$. The rate of decay is independent of the presence of tryptophan (as pure D or L) (Figure 4).

The MCD/EPR data (Figure 5) show that the substrate free enzyme is a mixture of low spin high spin six coordinate species, consistent with the water molecule seen in the crystal structure. When L-tryptophan is added to PrnB, the spectrum shows formation of a low-spin species. MCD/EPR indicates that this is six-coordinate with a nitrogenous ligand in the vacant coordination site. The addition of D-tryptophan shows oxygen atom coordination similar to but distinct from native (Figure 5).

\section{Discussion}

In crude extract, PrnB reacts with both 7-Cl-D- and 7-Cl-L-tryptophan almost equally as well. They could be equivalent substrates or it could be that the crude extracts contain a racemase which rapidly interconverts the D-enantiomer into the L-enantiomer. This would be inconsistent with the finding that from a mutant lacking PrnB only 7-Cl-L-tryptophan (no 7-CL-D-tryptophan) could be isolated (22). The fact that PrnB is inactivated by dialysis and purification by Ni-chelating chromatography, but can be re-activated by the addition of cellfree crude extract from the host strain $P$. fluorescens BL915 $\Delta$ orf1-4 indicates that a yet unknown co-factor or chemical / enzymatic transformation of substrate is required for PrnB 
activity. Simply adding reducing agents such as ascorbic acid has not led to activity recovery.

The topology of the core helical domain of PrnB is very similar to that of both IDO (and TDO) and the heme binding site is located in approximately the same position in all structures. Both PrnB and IDO are monomers and contain the small N-terminal domain which helps to form the heme binding pocket. This degree of similarity would point to a functional relationship, at least for the key catalytic step, between IDO / TDO and PrnB. IDO and PrnB structures are clearly more similar and the "disordered loop" at the heme site of PrnB is also disordered in IDO. TDO lacks the small N-terminal domain but rather another monomer contributes an a helix to the heme binding pocket, requiring at least a dimeric unit for activity; in fact, TDO is a tetramer. The "disordered loop" in PrnB is ordered in TDO and partly occludes the heme binding site. In our PrnB structures, the sixth site of the heme is ligated by the substrate, either by the amino nitrogen (L-tryptophan and 7-Cl-L-tryptophan) or the carboxyl group (D-tryptophan and 7-Cl-D-tryptophan). This is clearly inconsistent with the PrnB functioning as tryptophan 2,3 dioxygenase as this requires that $\mathrm{O}_{2}$ binds at $\mathrm{Fe}^{2+}$ not tryptophan. Of course a static crystal structure may be misleading. However the $\mathrm{pH}$ dependence of L-tryptophan and 7-Cl-L-tryptophan binding are consistent with their complex crystal structures (Figure S5). Further MCD and EPR studies of PrnB in solution indicate nitrogen ligation to the $\mathrm{Fe}^{3+}$ when L-tryptophan is added. The same experiments show oxygen ligation to the $\mathrm{Fe}^{3+}$ in D-tryptophan, thus the crystal structures are supported by solution measurements. In three complexes (D-tryptophan, 7-Cl-D-tryptophan and 7-Cl-L-tryptophan) the indole rings superimpose. We predict that this is indole binding site in a catalytically competent complex. In the absence of a dramatic conformational change, there is no other pocket which could accommodate a chlorinated indole ring. The binding mode observed in L-tryptophan would require significant conformation change to avoid clashes for any chlorinated indole and we believe the indole ring location in the Ltryptophan complex is a non-catalytic binding mode for L-tryptophan only.

We have been unable to detect any IDO or TDO activity from isolated PrnB using assays previously employed for IDO (12). Redox potential measurements also point towards PrnB being incapable of functioning as tryptophan 2,3 dioxygenase. The redox potential of the holo PrnB is $-109 \mathrm{mV}$ which is different than that of IDO, whose potential in the substrate free form was reported as $-30 \mathrm{mV}$ (23). Most strikingly in IDO the presence of L-tryptophan increases the redox potential to $+14 \mathrm{mV}$, stabilizing the $\mathrm{Fe}^{2+}$ state, whereas for PrnB, Ltryptophan significantly destabilizes the $\mathrm{Fe}^{2+}$ state $(-243 \mathrm{mV})$ and D-tryptophan even more so $(-280 \mathrm{mV})$. This is consistent with both D-tryptophan and L-tryptophan providing a sixth ligand and stabilizing to $\mathrm{Fe}^{3+}$ in solution. Thus in complete contrast to IDO, tryptophan deactivates PrnB towards 2,3 dioxygenase chemistry. Comparing the TDO and PrnB Ltryptophan complexes shows the indole rings are oriented in a similar manner with respect to the heme but shifted by over $2 \AA$ (Figure 2 ). In TDO, the amino nitrogen and carboxyl group of L-tryptophan are rotated and do not bind to the $\mathrm{Fe}^{3+}$. L-Tryptophan cannot adopt the conformation seen for TDO in PrnB, as F201 of PrnB would clash with the C $\beta$ of Ltryptophan. Similarly H55 of IDO would prevent the orientation of any of the tryptophan rings seen in PrnB (Figure 2). Structurally as well as biochemically, PrnB does not appear to posses IDO chemistry having an altered substrate binding pocket.

The structural similarity of PrnB to IDO (and TDO) does however argue that the enzymes are related mechanistically at some level. Comparing the L-tryptophan complexes suggests that PrnB shares a similar indole binding pocket. However, the other three complexes of PrnB suggest this pocket has been adapted to favor binding of the larger chlorinated substrates. Other changes in the active site have resulted in a significant change in redox potential at the heme and in the orientation of the tryptophan at the iron centre. The defining 
chemistry of IDO is the formation of the oxyferrous species and its insertion into the indole ring to form the iron tryptophan hydroperoxide. IDO has an oxyanion hole (centered on N125) close to the iron centre which could serve to stabilize the oxyferrous complex (12). PrnB has a similar oxyanion hole, although its precise orientation with respect to the heme is subtly different (Figure 2) and may explain the stability of this complex in PrnB. We propose that the mechanism for PrnB has retained the defining chemical step (Scheme 2) of peroxide insertion at the $\mathrm{CD} 2$ atom of tryptophan. In support of this we note that despite the differences in orientation between 7-Cl-L-tryptophan in PrnB and L-tryptophan in IDO (12) (Figure 2), the respective CD2 atoms are within $1.5 \AA$ of each other when the heme groups are superimposed. Our $\mathrm{Fe}^{3+}$ complexes therefore cannot be completely accurate representations of a productive complex, as the $\mathrm{Fe}^{2+}$ must be free to bind $\mathrm{O}_{2}$ and not be ligated to substrate. We propose that the amino (and carboxyl group) bound to the heme must rotate around the $\mathrm{CaC} \beta$ bond of tryptophan to free the sixth site to iron whilst the indole ring is fixed in position. We note that in the 7-Cl-L-tryptophan and 7-Cl-Dtryptophan complexes, the main chain atoms are rotated relative to each other whilst the chlorinated indole rings are in identical positions.

The difference between PrnB and IDO/TDO we believe is in the fate of the iron tryptophan hydroperoxide intermediate. PrnB does have a different architecture on the distal face to TDO / IDO. This is mirrored in its different redox potential and in differences in the indole binding site. These differences, we propose, prevent it from stabilizing the Criegee intermediate and stop tryptophan dioxygenase chemistry. In looking at the chemistry catalyzed by PrnB, the most likely intermediate would possess three fused rings (Scheme 3) and in fact chemical studies on peroxy tryptophan have shown that such a tricyclic species does form (24). The three ring structure has precedence in biomimetic organic chemistry (pyrrolobenzoxazine core unit of paeciloxazine (25) Scheme 3) and most significantly in other chlorinated and non-chlorinated natural products (pyrroindomycin A/B, scheme 3) (26). We suggest that PrnB catalyses the formation of a three fused ring intermediate (as opposed to the Criegee intermediate) by utilizing the electron withdrawing properties of the tryptophan hydroperoxide intermediate to activate the indole ring for nucleophilic attack (Scheme 4). The thee ring system can re-arrange (Scheme 4) by the reversible elimination of the peroxide. An irreversible elimination step would ultimately decide the fate of the three ring intermediate. This mechanism would give rise to three possibilities: the pyrrolnitrin like product (loss of $\mathrm{CO}_{2}$ ): the pyrroindomycin (26) like product (loss of $\mathrm{H}_{2}$ ): an arylpyrrole compound (Scheme 4). Although arylpyrrole compounds are well described in chemistry (27) they are not well known natural products. However, the arylpyrrole derivative, 2carboxy-4-(2' -amino- $3^{\prime}$-chlorophenyl)pyrrole as well as its bromo analogue (Scheme 3) have been isolated from the pyrrolnitrin producer $P$. aureofaciens $(28,29)$. The fact that all three possible chlorinated products that could result from the iron hydroperoxide three ring intermediate are found in nature, we take as support for our mechanistic hypothesis. We have considered an alternative mechanism (Supporting material) involving proton abstraction from the $\mathrm{Ca}$ atom and $\mathrm{N}$ atom of 7-Cl-L-tryptophan. However there are no appropriate residues located close to these atoms and such a mechanism would involve an $\mathrm{Fe}^{4+}$ species.

Although enzyme activity in isolated extracts is robust, a more accurate delineation of the mechanism requires we obtain activity under defined conditions. We can only speculate on the lack of activity of the purified enzyme. Our protocols could inactivate the protein in some way related to the redox conditions (loss of co-factor or reducing enzyme). Alternatively, there is an additional specific essential enzyme which complexes with PrnB or modifies substrate. The conservation of the four gene cluster and its successful insertion into plants would seem to make it very unlikely there is a specific PrnB partner enzyme or specific 7-Cl-tryptophan modifying enzyme. Despite this, PrnB is a significant addition to 
the IDO/TDO superfamily. The involvement of a dioxygenase heme in this novel indole rearrangement is surprising. Our work suggests there may be more members of this superfamily to be discovered which catalyze related but different indole chemical modifications. These putative enzymes could well have important synthetic applications. Our investigations into this fascinating class of enzyme will continue.

\section{Supplementary Material}

Refer to Web version on PubMed Central for supplementary material.

\section{Acknowledgments}

We thank Ms. M. Thorke, Biochemie, TU Dresden, for synthesis of racemic 7-chlorotryptophan and Ms. A. Ernyei, Biochemie TU Dresden, for enzymatic preparation of enaniomerically pure D- and L-7-Cl-tryptophan. We thank Dr R. Goss University of East Anglia for pure L-7-Cl-tryptophan. We are also indebted to Myles Cheesman, University of East Anglia, for MCD and EPR assistance and Sarah Thackray, University of Edinburgh, for help with OTTLE experiments.

\section{References}

(1). Chernin L, Brandis A, Ismailov Z, Chet I. Pyrrolnitrin production by an Enterobacter agglomerans strain with a broad spectrum of antagonistic activity towards fungal and bacterial phytopathogens. Curr. Microbiol. 1996; 32:208-212.

(2). Gerth K, Trowitzsch W, Wray V, Hofle G, Irschik H, Reichenbach H. Pyrrolnitrin from Myxococcus-Fulvus (Myxobacterales). J. Antibiot. 1982g; 35:1101-1103. [PubMed: 6815147]

(3). Kalbe C, Marten P, Berg G. Strains of the genus Serratia as beneficial rhizobacteria of oilseed rape with antifungal properties. Microbiol. Res. 1996; 151:433-439. [PubMed: 9022304]

(4). Smilanick JL, Denisarrue R. Control of Green Mold of Lemons with Pseudomonas Species. Plant Disease. 1992; 76:481-485.

(5). Jespers ABK, Dewaard MA. Natural-Products in Plant-Protection. Netherlands Journal of Plant Pathology. 1993; 99:109-117.

(6). Ligon JM, Hill DS, Hammer PE, Torkewitz NR, Hofmann D, Kempf HJ, van Pee KH. Natural products with antifungal activity from Pseudomonas biocontrol bacteria. Pest Manage. Sci. 2000; 56:688-695.

(7). Tripathi RK, Gottlieb D. Mechanism of Action of the Antifungal Antibiotic Pyrrolnitrin. J. Bacteriol. 1969; 100:310-318. [PubMed: 4310080]

(8). Kirner S, Hammer PE, Hill DS, Altmann A, Fischer I, Weislo LJ, Lanahan M, van Pee KH, Ligon JM. Functions encoded by pyrrolnitrin biosynthetic genes from Pseudomonas fluorescens. J. Bacteriol. 1998; 180:1939-1943. [PubMed: 9537395]

(9). Dong CJ, Flecks S, Unversucht S, Haupt C, van Pee KH, Naismith JH. Tryptophan 7-halogenase (PrnA) structure suggests a mechanism for regioselective chlorination. Science. 2005; 309:22162219. [PubMed: 16195462]

(10). Yeh E, Blasiak LC, Koglin A, Drennan CL, Walsh CT. Chlorination by a long-lived intermediate in the mechanism of flavin-dependent halogenases. Biochemistry. 2007; 46:1284-1292. [PubMed: 17260957]

(11). Lee JK, Ang EL, Zhao HM. Probing the substrate specificity of aminopyrrolnitrin oxygenase (PrnD) by mutational analysis. J. Bacteriol. 2006; 188:6179-6183. [PubMed: 16923884]

(12). Forouhar F, Anderson JLR, Mowat CG, Vorobiev SM, Hussain A, Abashidze M, Bruckmann C, Thackray SJ, Seetharaman J, Tucker T, Xiao R, Ma L-C, Zhao L, Acton TB, Montelione GT, Chapman SK, Tong L. Molecular insights into substrate recognition and catalysis by tryptophan 2,3-dioxygenase. Proc. Natl. Acad. Sci. USA. 2007; 104:473-478. [PubMed: 17197414]

(13). Zhang Y, Kang SA, Mukherjee T, Bale S, Crane BR, Begley TP, Ealick SE. Crystal Structure and Mechanism of Tryptophan 2,3-Dioxygenase, a Heme Enzyme Involved in Tryptophan Catabolism and in Quinolinate Biosynthesis. Biochemistry. 2007; 46:145-155. [PubMed: 17198384] 
(14). Sugimoto H, Oda S.-i. Otsuki T, Hino T, Yoshida T, Shiro Y. Crystal structure of human indoleamine 2,3-dioxygenase: Catalytic mechanism of $\mathrm{O} 2$ incorporation by a heme-containing dioxygenase. Proc. Natl. Acad. Sci. USA. 2006; 103:2611-2616. [PubMed: 16477023]

(15). De Laurentis W, Leang K, Hahn K, Podemski B, Adam A, Kroschwald S, Carter LG, van Pee KH, Naismith JH. Preliminary crystallographic characterization of PrnB, the second enzyme in the pyrrolnitrin biosynthetic pathway. Acta Crystallogr., Sect. F: Struct. Biol. Cryst. Commun. 2006; 62:1134-1137.

(16). Lee MS, Phillips RS. Enzymatic-Synthesis of Chloro-L-Tryptophans. Bioorg. Med. Chem. Lett. 1992; 2:1563-1564.

(17). Murshudov GN, Vagin AA, Dodson EJ. Refinement of macromolecular structures by the maximum-likelihood method. Acta Crystallogr., Sect. D: Biol. Crystallogr. 1997; 53:240-255. [PubMed: 15299926]

(18). Aasa R, Vanngard T. EPR Signal Intensity and Powder Shapes - Re-Examination. J. Magn. Reson. 1975; 19:308-315.

(19). Ost TWB, Clark JP, Anderson JLR, Yellowlees LJ, Daff S, Chapman SK. 4-Cyanopyridine, a versatile spectroscopic probe for cytochrome P450BM3. J. Biol. Chem. 2004; 279:48876-48882. [PubMed: 15364917]

(20). Krissinel E, Henrick K. Detection of protein assemblies in crystals. Computational Life Sciences, Proceedings. 2005; 3695:163-174.

(21). Binkowski TA, Naghibzadeh S, Liang J. CASTp: Computed atlas of surface topography of proteins. Nucleic Acids Res. 2003; 31:3352-3355. [PubMed: 12824325]

(22). Hohaus K, Altmann A, Burd W, Fischer I, Hammer PE, Hill DS, Ligon JM, van Pee KH. NADH-dependent halogenases are more likely to be involved in halometabolite biosynthesis than haloperoxidases. Angew. Chem., Int. Ed. 1997; 36:2012-2013.

(23). Papadopoulou ND, Mewies M, McLean KJ, Seward HE, Svistunenko DA, Munro AW, Raven EL. Redox and Spectroscopic Properties of Human Indoleamine 2,3-Dioxygenase and A His303Ala Variant: Implications for Catalysis. Biochemistry. 2005; 44:14318-14328. [PubMed: 16245948]

(24). Nakagawa M, Watanabe H, Kodato S, Okajima H, Hino T, Flippen JL, Witkop B. Valid Model for Mechanism of Oxidation of Tryptophan to Formylkynurenine - 25 Years Later. Proc. Natl. Acad. Sci. USA. 1977; 74:4730-4733. [PubMed: 16592461]

(25). Schwaebisch D, Tchabanenko K, Adlington RM, Cowley AM, Baldwin JE. Biomimetic synthesis of the pyrrolobenzoxazine core of paeciloxazine. Chem. Commun. 2004:2552-2553.

(26). Ding WD, Williams DR, Northcote P, Siegel MM, Tsao R, Ashcroft J, Morton GO, Alluri M, Abbanat D, Maiese WM, Ellestad GA. Pyrroindomycins, Novel Antibiotics Produced by Streptomyces-Rugosporus Sp L1-42d005w .1. Isolation and Structure Determination. J. Antibiot. 1994; 47:1250-1257. [PubMed: 8002387]

(27). Pinna GA, Loriga G, Murineddu G, Grella G, Mura M, Vargiu L, Murgioni C, La Colla P. Synthesis and anti-HIV-1 activity of new delavirdine analogues carrying arylpyrrole moieties. Chem. Pharm. Bull. 2001; 49:1406-1411. [PubMed: 11724230]

(28). Salcher O, Lingens F, Fischer P. Biosynthesis of Pyrrolenitrine - Determination of 4-(2' Amino-3' -Chlorophenyl)Pyrrole-2-Carboxylic Acid. Tetrahedron Lett. 1978:3097-3100.

(29). van Pee KH, Salcher O, Fischer P, Bokel M, Lingens F. The Biosynthesis of Brominated Pyrrolnitrin Derivatives by Pseudomonas-Aureofaciens. J. Antibiot. 1983; 36:1735-1742. [PubMed: 6662814] 

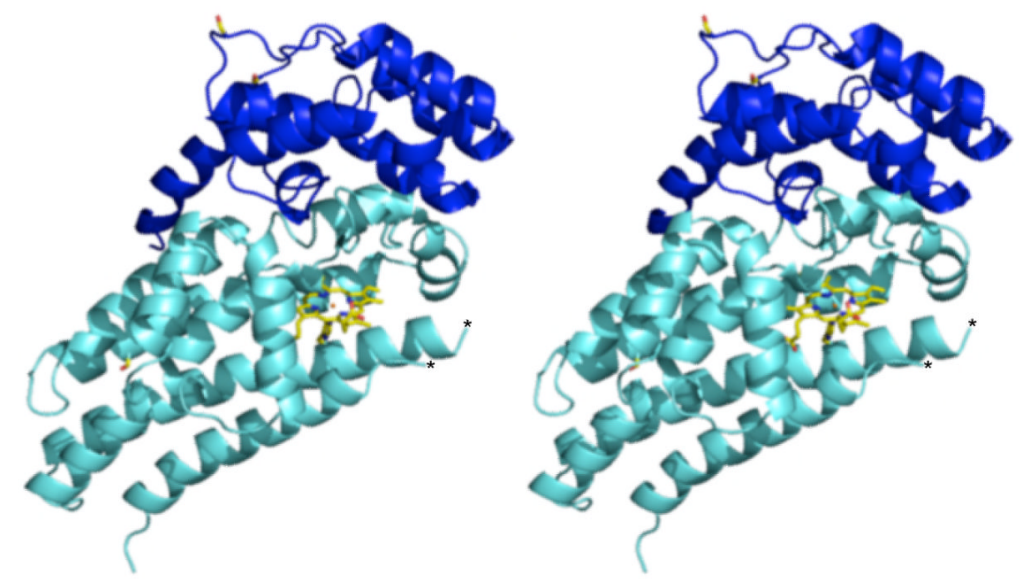

Figure 1.

A stereo ribbon representation of the structure of $\operatorname{PrnB}$, the core helical domain is colored cyan and the N-terminal capping domain is colored blue. The cyan colored helices are found in TDO, the N-terminal domain is not. The disordered loop is marked by a * at each end. The side chains of three cysteine residues (20,60 and 175) which were mutated to serine are shown in sticks. The heme group and the proximal H313 ligand are shown in sticks with carbon yellow, nitrogen blue and oxygen red. 
a

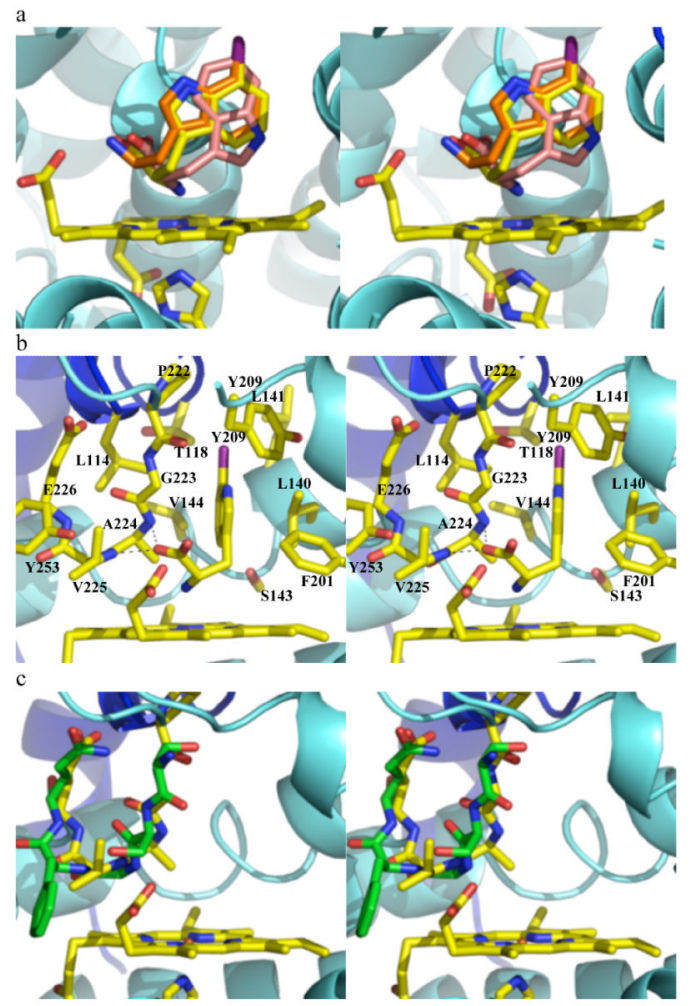

d

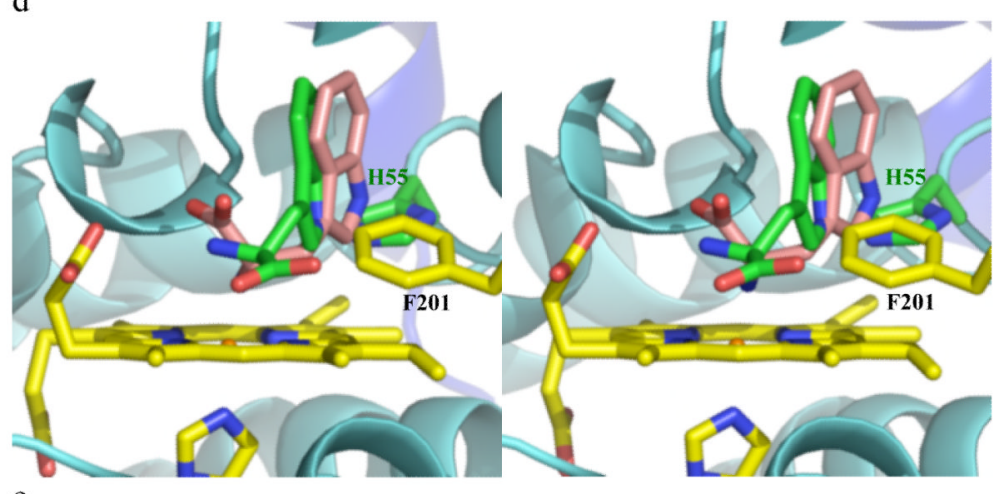

e

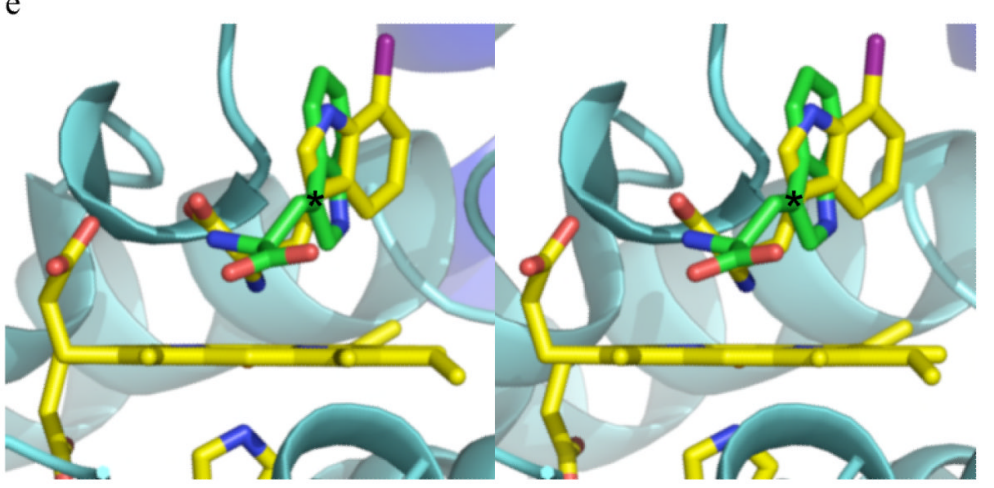

Figure 2. 
(a) The complexes of PrnB with substrates 7-Cl-L-tryptophan, 7-Cl-D-tryptophan and Ltryptophan (D-tryptophan overlaps with 7-Cl-D-tryptophan and is not shown). The protein is colored as Figure 1, non carbon atoms are colored as Figure 1, chlorine atoms are purple, for 7-Cl-L-tryptophan carbon atoms are colored yellow, carbons in 7-Cl-D-tryptophan are colored orange and carbons in L-tryptophan are colored salmon. Although the chloro indole rings of 7-Cl-L-tryptophan and 7-Cl-D-tryptophan overlap, the main chain atoms do not. Ltryptophan binds in a different manner to the other complexes, although the part of the indole rings overlap. The proximal ligand to the heme (H313) is shown. In 7-Cl-L-

tryptophan and L-tryptophan, the amino nitrogen atom ligates to the iron, whereas in 7-ClD-tryptophan one of the carboxyl oxygen atoms ligates to the iron. (b) The active site of PrnB. The residues that surround the 7-Cl-L-tryptophan ligand are shown. The oxyanion hole at A224 is labeled and the hydrogen bonds shown. Atoms are colored as Figure 2a. (c) The subtle differences in the active sites of PrnB (colored as Figure 2a) and TDO(carbon atoms green, other atoms colored as Figure $2 \mathrm{a}$ ). The superposition is based on the heme groups of each protein, the different orientation of the (d) Superposition of the L-tryptophan complexes of PrnB and TDO (PDB code 2NW8); atoms colored as Figure 2c. The binding sites are mutually exclusive, F201 in PrnB prevents L-tryptophan binding as seen in TDO, whilst H55 of TDO prevents any of the binding modes for tryptophan seen in PrnB. (e) The substrate complexes of PrnB and TDO show that despite quite different orientations the CD2 atom (marked with $*$ ) where the peroxide is predicted to be inserted are similarly positioned relative to the heme. 

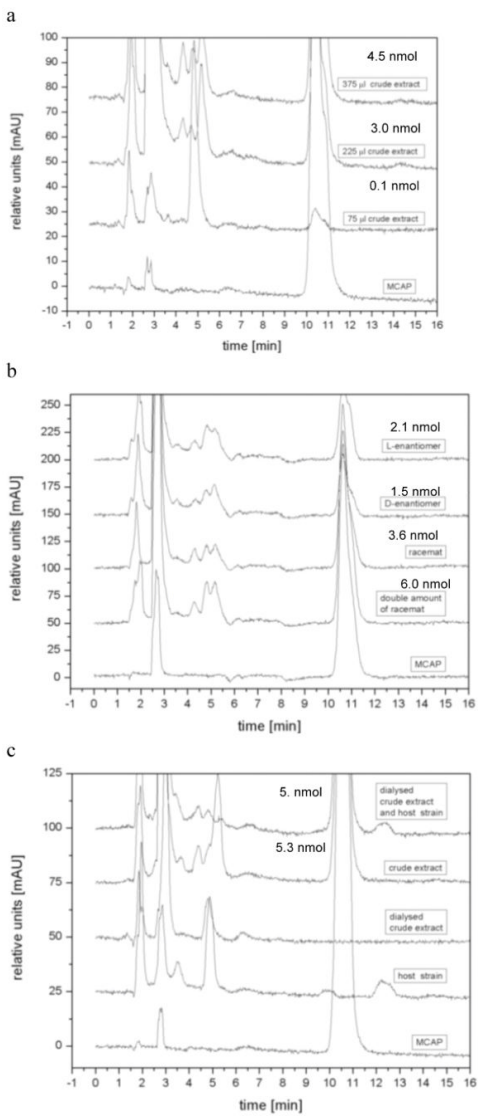

Figure 3.

(a) HPLC-analysis of the in vitro activity assay of PrnB incubated with racemic 7chlorotryptophan. The bottom line shows monodechloroaminopyrrolnitrin (MCAP) the product formed by PrnB from 7-chlorotryptophan as a standard. The other lines show from bottom to top increasing volume of crude extracts. The numbers on the right hand side give the amount of MCAP corresponding to the peak. (b) HPLC-analysis of the in vitro activity assay of PrnB incubated with racemic 7-chlorotryptophan and the pure D- and Lenantiomers, respectively. The lines from bottom to top show: MCAP standard; incubation with racemic 7-chlorotryptophan $(0.5 \mathrm{mM}$ and $0.25 \mathrm{mM}$ final concentration, respectively); incubation with $0.5 \mathrm{mM}$ 7-chloro-D-tryptophan; incubation with $0.5 \mathrm{mM} 7-\mathrm{Cl}-\mathrm{L}$-tryptophan. (c) HPLC-analysis of in vitro activity assays of PrnB. The lines from bottom to top show: MCAP as the standard; crude extract of the host strain P.fluorescens BL915 $\Delta$ orf1-4; dialyzed PrnB-containing extract; cell-free crude extract containing PrnB; restoration of PrnB activity by incubation of dialyzed crude extract together with non-dialyzed crude extract of the host strain. 

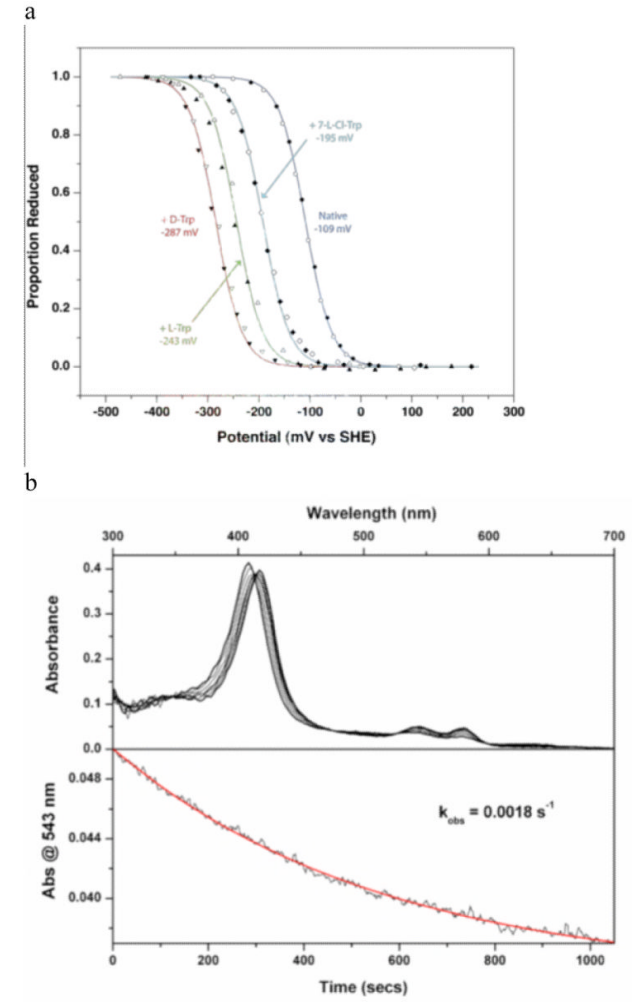

Figure 4.

(a) OTTLE potentiometry of PrnB in the absence of L-Tryptophan (circles), $+20 \mathrm{mM} \mathrm{L-}$ Tryptophan (triangles pointing up), $+20 \mathrm{mM}$ D-Tryptophan (triangles pointing down) and $10 \mathrm{mM}$ 7-Chloro-L-Tryptophan. In each case the filled and empty shapes correspond to the reductive and oxidative traces respectively. Each data set is fit using the Nernst equation and the fit is coloured according to the corresponding label. (b) Decay of oxyferrous PrnB. Upper Panel. Spectral changes associated with the decay of the oxyferrous PrnB species. Lower Panel. Exponential decrease in absorbance at $543 \mathrm{~nm}$ used to determine the rate of oxyferrous PrnB decay. Red line indicates single exponential 


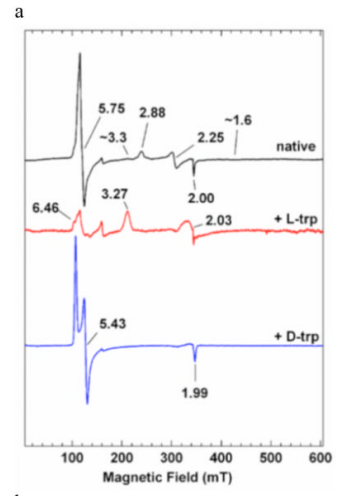

b
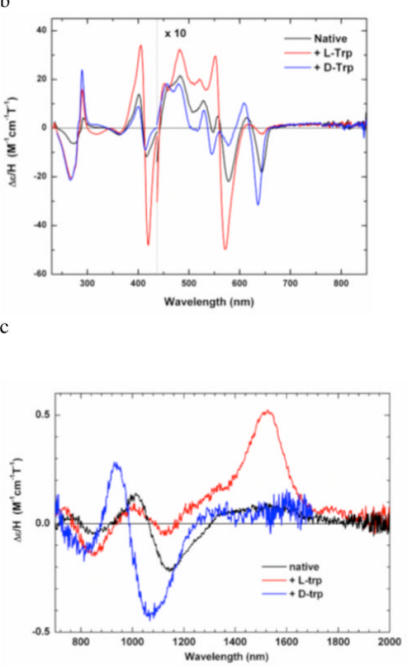

Figure 5.

(a) (a) Perpendicular mode X-band EPR spectra of native ferric PrnB in the absence of tryptophan (black line), ferric PrnB + $20 \mathrm{mM}$ L-tryptophan (red line) and ferric PrnB + 20 mM D-tryptophan (blue line). Conditions: microwave frequency, $9.67 \mathrm{GHz}$; microwave power, $2 \mathrm{~mW}$; modulation amplitude, $10 \mathrm{G}$; temperature, $10 \mathrm{~K}$; scan speeds and time constants are the same for all spectra. Spectra have been adjusted for differences in the enzyme concentration and receiver gain where required. Gains were typically of the order of $2 \times 10^{5}-8 \times 10^{5}$. (b) Room-temperature MCD at $5 \mathrm{~T}$ in the UV/visible region of native ferric PrnB in the absence of tryptophan (black line), ferric PrnB $+20 \mathrm{mM}$ L-tryptophan (red line) and ferric PrnB + $20 \mathrm{mM}$ D-tryptophan (blue line). All spectra were recorded at $\mathrm{pH}$ 7.0. (c) Room-temperature MCD at $5 \mathrm{~T}$ in the near-IR region of native ferric PrnB in the absence of tryptophan (black line), ferric PrnB + $20 \mathrm{mM}$ L-tryptophan (red line) and ferric PrnB + 20 mM D-tryptophan (blue line). All spectra were recorded at pH 7.0 with $50 \mu \mathrm{M}$ enzyme. 


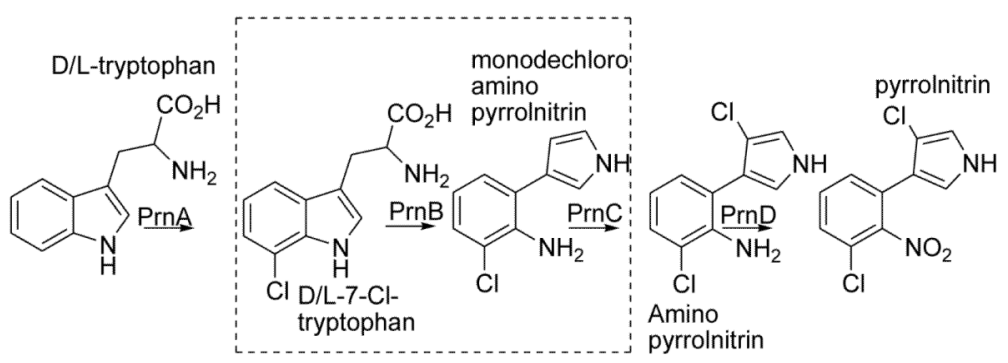

Scheme 1.

Pyrrolnitrin pathway. The reaction catalyzed by PrnB is boxed. 


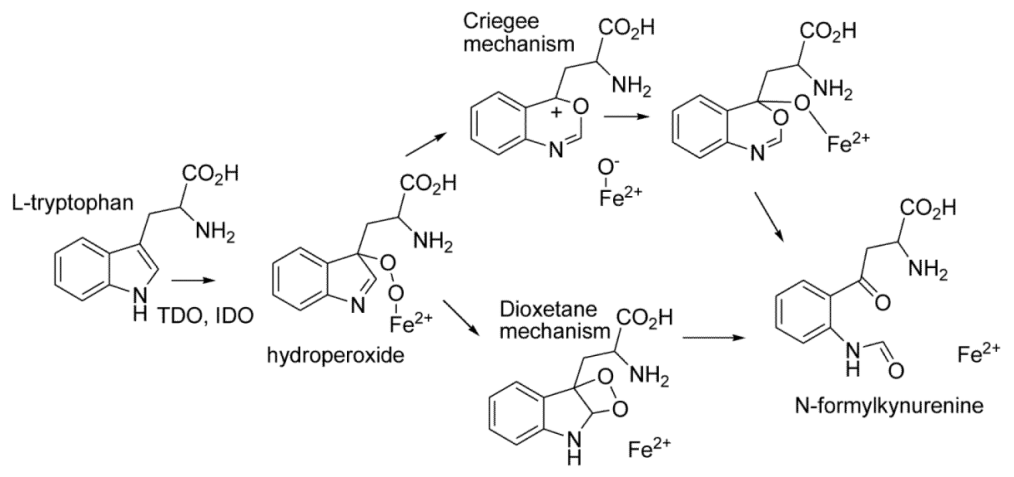

Scheme 2.

The reaction catalyzed by TDO and by IDO. 


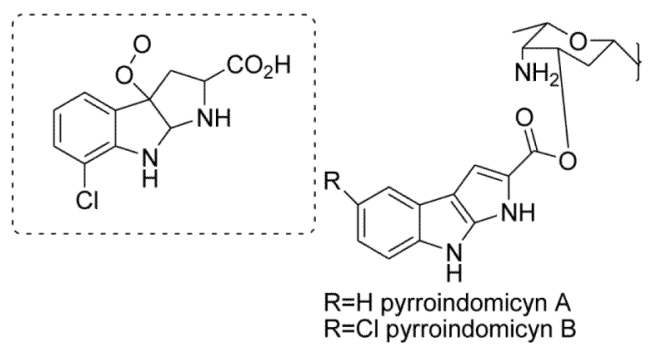

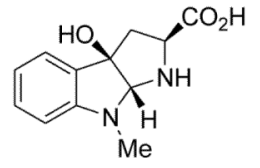

pyrrolobenzoxazine core

Scheme 3 .

The three fused ring intermediate proposed to form during PrnB catalysis. This has analogues in the natural product pyrroindomicyn (26) and biomimetic chemistry (pyrrolobenzoxazine core) (25), an arylpyrrol structure has been found in pyrrolnitrin producing bacteria $(28,29)$. 


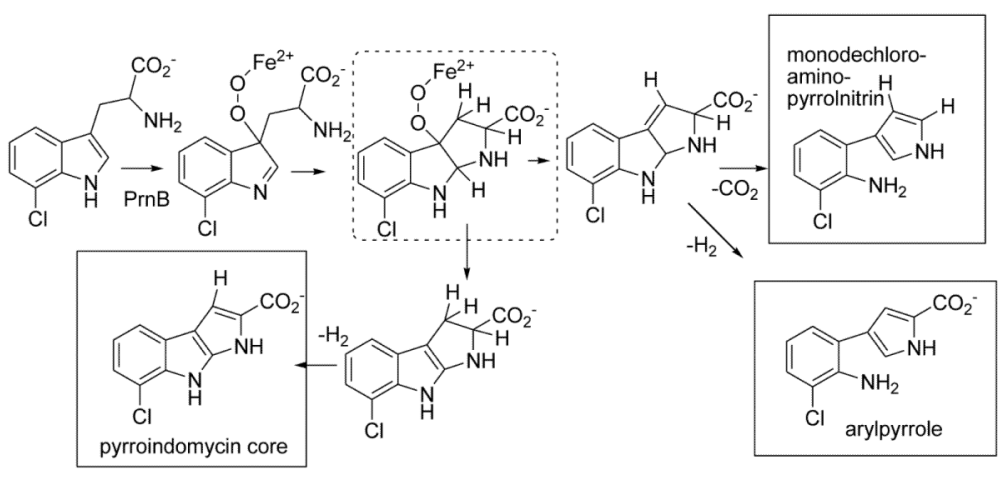

Scheme 4.

The proposed chemical route followed during PrnB catalysis, the tricyclic ring structure is boxed. A route to the pyrroindomycin core and ayrlpyrrole core structures are shown (24). 


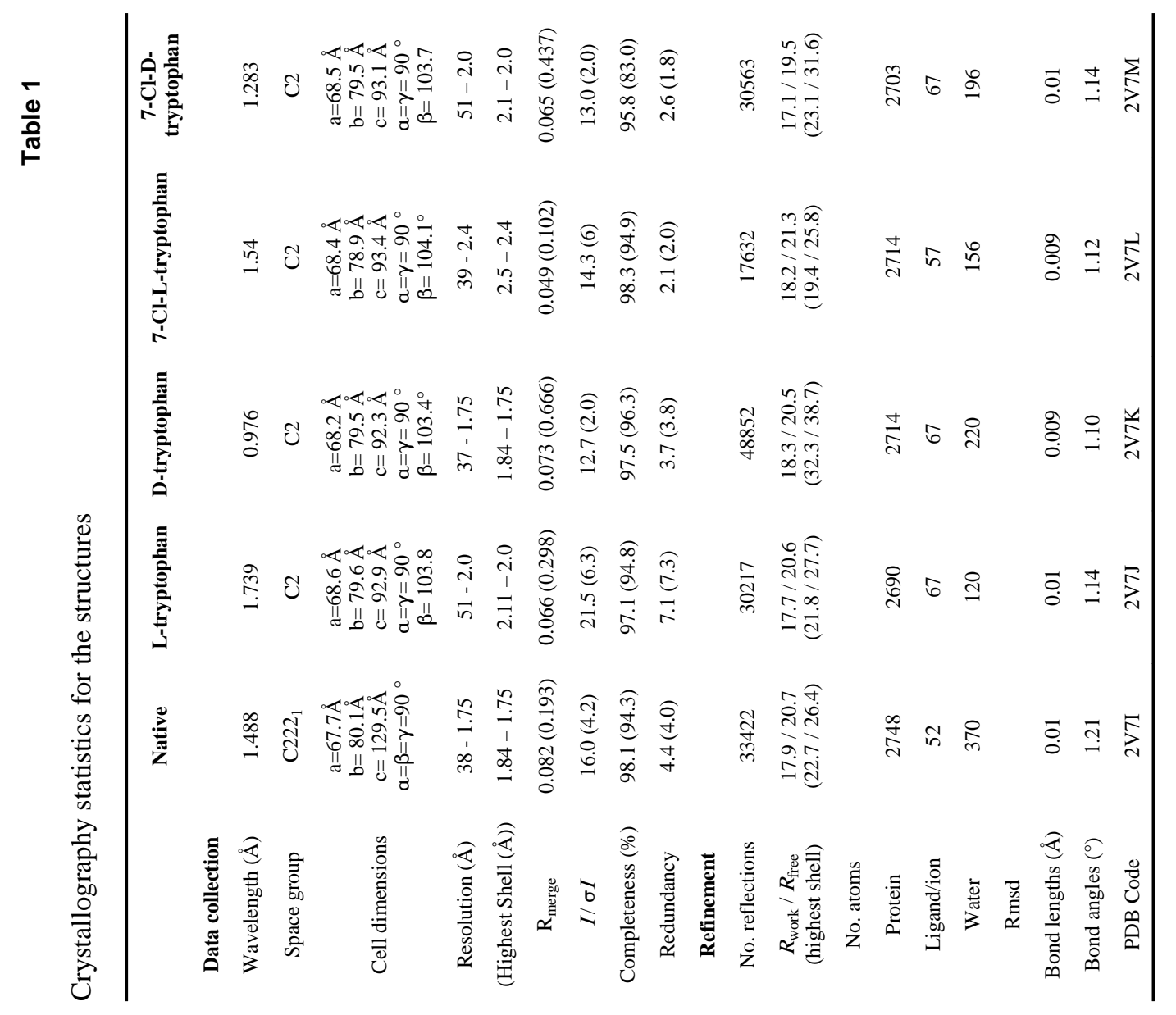

